\title{
Ki-67 status in patients with primary breast cancer and its relationship with other prognostic factors
}

\author{
Touraj Asvadi Kermani ${ }^{1}$, Iraj Asvadi Kermani ${ }^{2}$, Zhaleh Faham ${ }^{2}$, Roya Dolatkhah ${ }^{2}, *$
}

${ }^{I}$ Department of Surgery, Imam Reza Educational and Treatment Center, Tabriz University of Medical Sciences, Tabriz, Iran

${ }^{2}$ Hematology and Oncology Research Center, Tabriz University of Medical Sciences, Tabriz, Iran

Correspondence

Roya Dolatkhah, Hematology and Oncology Research Center, Tabriz University of Medical Sciences, Tabriz, Iran

Email: dolatkhahr@tbzmed.ac.ir

History

- Received: Nov 17, 2018

- Accepted: Jan 21, 2019

- Published: Feb 17, 2019

DOI :

https://doi.org/10.15419/bmrat.v6i2.520

\section{Check for updates}

\section{Copyright}

(c) Biomedpress. This is an openaccess article distributed under the terms of the Creative Commons Attribution 4.0 International license.

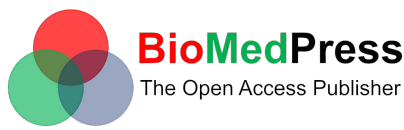

\begin{abstract}
Introduction: Breast cancer (BC) is the most common cancer in women and is the second most common cause of fatality in patients with cancer in the world. Cell proliferation plays an important role in the clinical behavior of invasive BC. We aimed to assess the status of Ki-67 in patients with primary breast cancer and evaluate the association of this tumor marker with other clinico-pathologic and prognostic factors. Methods: The current study recruited 220 patients with primary BC admitted to the oncology clinic of the Tabriz University of Medical Sciences. We evaluated Ki-67 IHC slides and reported the Ki-67 status and its relationship with other prognostic factors in breast cancer patients. Among 220 patients, 63.3\% developed grade 2 tumors, and 63.8\% were younger than 50-year-olds. 117 cases (53\%) were Ki-67 positive with more than 1\% tumor nuclei stained, and 53 cases (24\%) had tumors with more than $15 \%$ of Ki-67 expression. Results: There was no correlation between Ki-67 and patient's age (Spearman rho $=0.375$, tau Kendall $=0.374$ ), tumor size (Spearman rho $=0.558$, tau Kendall $=0.548$ ) and grade (Spearman rho $=0.570$, tau Kendall $=0.568$ ), however, there was a marginally significant relationship between lymph node status and Ki-67 expression (Spearman rho $=0.077$, tau Kendall $=0.079$ ). Based on the Mann -Whitney test, there was a significant correlation between the expression of estrogen receptor (ER) and progesterone receptor (PR) with Ki-67. Conclusion: A reliable estimation of different prognostic factors in $\mathrm{BC}$ patients is required for the selection of an optimal therapeutic strategy. The attention has been focused on the markers of tumor biology.
\end{abstract}

Key words: Breast Cancer, Grade, Lymph Node, Prognosis, Tumor Markers

\section{INTRODUCTION}

Breast cancer (BC) is the most common cancer and the second most common cause of death in women. Globally, one in 14 women will develop breast cancer between the age of $0-79$, which comes down to 1 in 9 women in the developed countries ${ }^{1}$. Breast cancer is the most common cancer among Iranian women and ranked the $5^{\text {th }}$ leading cause of cancer death in women ${ }^{2}$.

Prognostic factors are essential in BC diagnosis as they allow the identification of high-risk patients, for whom, an adjuvant therapy can improve prognosis ${ }^{3,4}$. The traditional prognosis can only identify the group of approximately $30 \%$ of patients and their outcome. Therefore, new prognostic markers are required ${ }^{5,6}$. Considering the fact that radiotherapy and different medical hormonal manipulations may develop side effects in patients, the risk-based refined approaches are essential to reduce these side effects. Some new prognostic factors have been described over the last few years ${ }^{7}$. However, most of them still require clinical validation $^{8,9}$.

In exploration for the potential prognostic indicators of breast cancer, attention has been focused on tumor markers. Cell proliferation plays an important role in the clinical behavior of invasive BC. Increased cell proliferation is correlated with poor prognosis. Ki-67 labeling index is more sensitive than other techniques such as mitotic figure counts because cells with active phases of the cell cycle can be recognized; furthermore, a reliable assessment of mitotic figures is more time-consuming than the counting of nuclei in immunohistochemistry (IHC). The reproducible mitotic index is not usually obtained without special training in counting with fraction assessed method, yet mitotic count and Ki-67 index are still considered to be the most practical methods ${ }^{10,11}$.

Interest in $\mathrm{Ki}-67$ has recently increased as $\mathrm{Ki}-67$ is a potential marker for predicting the responsiveness to chemotherapy ${ }^{12}$. Compared with other markers, Ki67 immuno-staining is a convenient method for assessing the proliferating index. Ki-67 IHC is a rapid and inexpensive technique that can be easily used in almost all pathological laboratories and requires only a small tissue sample, including those obtained from fine-needle aspirations. Ki-67 levels are known to be associated with positive prognosis in most studies $^{13,14}$. 
In this cross-sectional analytical study, we aimed to assess the status of Ki-67 in patients with primary breast cancer and evaluate the association of Ki-67 with other factors, including patient's age, lymph node status, ER, PR, and the grade and size of tumors. We demonstrate that the association of the tumor biological marker, Ki-67, with other clinicopathologic aspects is a simple and cost-effective test to manage the patients' response to treatment and their outcome.

\section{METHODS}

This study included 220 patients with primary BC admitted to the oncology clinic of Tabriz University of Medical Sciences. The data of this analyticaldescriptive study were obtained from patient's documents in this clinic. The levels of ER, PR, Ki-67, LN status, and the tumor grades and sizes were determined after diagnosis. Our pathologist reevaluated all smearing stain of Ki- 67 by IHC, and the exact levels of $\mathrm{Ki}-67$ were determined. The grade of tumors was confirmed by an expert pathologist, and the lymph node status was confirmed clinically using imaging techniques after surgery.

\section{IHC analysis}

IHC staining for ER, PR, and Ki-67 was performed in all cases. The sample sections were de-paraffinized in alcohol and xylene and then heated in EDTA buffer solution $(\mathrm{PH}=9)$ to $100^{\circ} \mathrm{C}$. After cooling for about 15 minutes, the samples were rinsed in tris buffer solution $(\mathrm{PH}=7.6)$ for 5 minutes. Endogenous peroxidase was quenched with $3 \%$ hydrogen peroxidase in methanol to block nonspecific binding for 10 minutes. The slides were then incubated for 30 minutes with primary antibodies. The primary antibodies used for estrogen receptor was ER, Dako, clone ID 5, for progesterone receptor was PR, Dako, clone PgR636, and for Ki-67 was Dako, clone MIB-1. The tumor grade was reported based on the H\&E (hematoxylin and eosin) smears, which were considered in 3 parameters: the number of mitoses, the nuclear polymorphism rates, and the gland formation structures. If the total score of them were $3-5,6-7$, and $8-9$, we considered the grades as 1,2 , and 3 respectively. The ER, PR, and Ki-67 status were defined based on the intensity and the percentage of nuclear stain. Negative Ki-67 was defined as less than $1 \%$ stain, and positive $\mathrm{Ki}-67$ was greater than $1 \%$. Patients with positive Ki67 were divided into 3 groups: $1-5 \%, 6-14 \%$ and $\geq$ $15 \%$ of Ki-67 staining ${ }^{15,16}$.

\section{Statistical analysis}

We used the Mann-Whitney U test to determine the correlation between Ki-67 and ER and PR expression because the goal was to evaluate the relationship between an ordinal variant Ki-67 and two unpaired sample variants, ER and PR. To determine the relationship between patient's age, tumor size and grade, and LN invasion in patients with primary BC, Spearman's rho and the Kendall rank correlation coefficient tests were used. SPSS 21 Statistical Analysis Software (SPSS Inc., Chicago, IL.) was used to perform all statistical analysis.

The study protocol was reviewed and confirmed by the ethic committee of Tabriz University of Medical Sciences (confirmed ID: 5/4/9587). Written consent forms were provided to all patients enrolled in the study, and all patients' data and information were confidential.

\section{RESULTS}

From the overall of 220 patients with confirmed breast cancer, $63.8 \%$ were younger than 50 years old. Ki67 was negative in $47 \%$ of the patients and positive in $53 \%$ of them. The levels of Ki-67 were respectively $1-5 \%, 6-14 \%$ and $\geq 15 \%$ in $18 \%, 11.1 \%$ and $24 \%$ of patients with positive Ki-67 (Figure 1). In terms of tumor size, $30.7 \%$ of patient's tumors were $\leq 2 \mathrm{~cm}$, $51.4 \%$ were $2-5 \mathrm{~cm}$, and the remainder $(17.9 \%)$ was larger than $5 \mathrm{~cm}$. The frequency of grade 1,2 and 3 tumors were respectively $23.9 \%, 63.3 \%$, and $12.8 \%$.

No significant relationship was found between Ki-67 and patient's age (Spearman rho $=0.375$, tau Kendall $=0.374$ ), tumor's size ( Spearman rho $=0.558$, tau Kendall $=0.548)$ and grade (Spearman rho $=0.570$, tau Kendall = 0.568).

Based on the lymph node (LN) status, patients were divided into 3 groups: no $\mathrm{LN}$ invasion, 1-3 LN, and more than $3 \mathrm{LN}$ invasion. The frequency of these groups in patients were respectively $41.6 \%, 19.6 \%$, and $38.8 \%$. No significant relationship was observed between LN status and Ki-67 status (The Spearman rho $=0.077$, tau Kendall $=0.079)($ Table 1$)$.

The examination of ER and PR showed that $60.1 \%$ of patients were ER-positive and 59.2\% were PR positive. To determine the correlation between Ki-67 levels and ER status in the patients, the Mann-Whitney $U$ test was performed and showed a significance of $P=0.048$. However, only a marginally significance was observed for PR and Ki-67 ( $P=0.056)$.

We also examined the relationship between lymph node status and Ki-67 levels in BC patients and found no significant relationship between them (spearman 


\section{Level of Ki67 biomarker as IHC staining in 220 breast cancer patients}

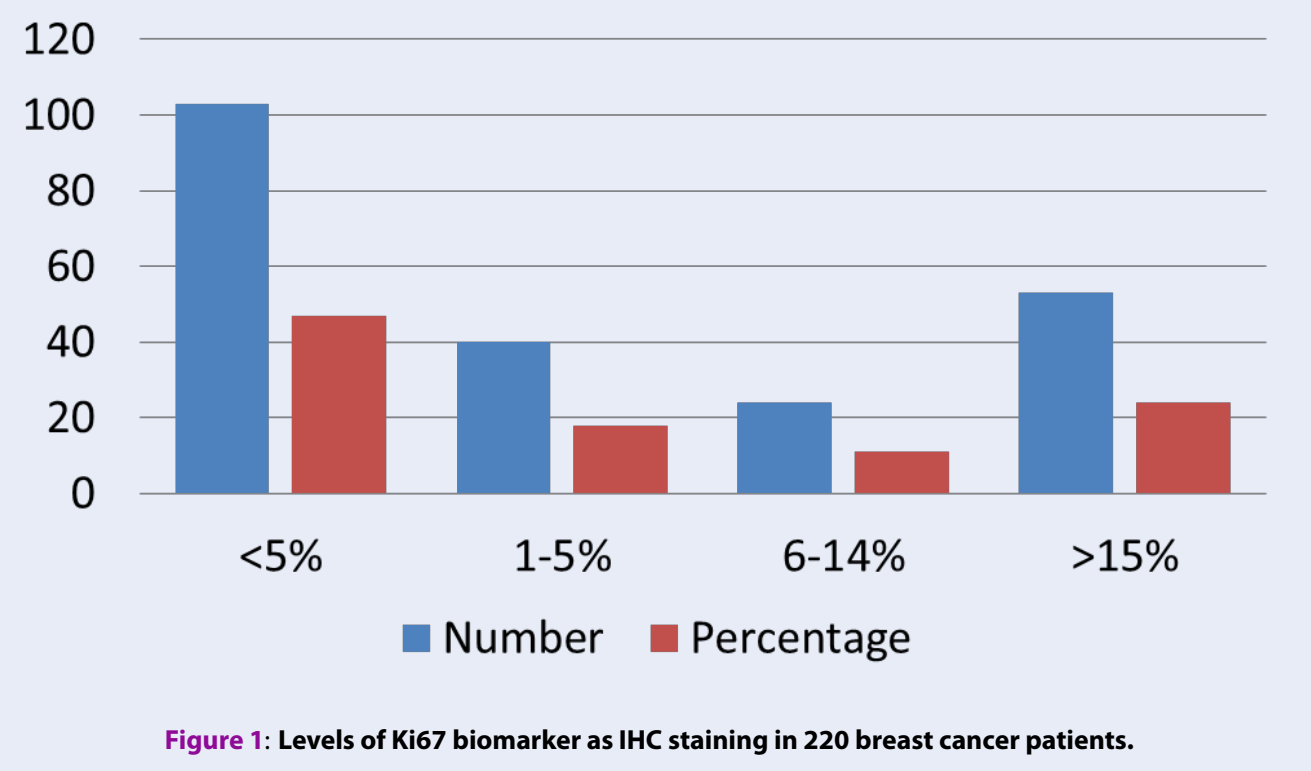

\begin{tabular}{|c|c|c|c|c|c|}
\hline Variables & & Number & Percentage (\%) & Spearman rho* & Tau Kendall ${ }^{\star}$ \\
\hline \multirow[t]{2}{*}{ Age } & $\leq 50$ & 140 & 63.8 & 0.375 & 0.374 \\
\hline & $>50$ & 80 & 36.2 & & \\
\hline \multirow[t]{3}{*}{ Tumor Size $(\mathrm{cm})$} & $\leq 2$ & 68 & 30.7 & 0.558 & 0.548 \\
\hline & $2-5$ & 113 & 51.4 & & \\
\hline & $>5$ & 39 & 17.9 & & \\
\hline \multirow[t]{3}{*}{ Grade } & I & 53 & 23.9 & 0.570 & 0.568 \\
\hline & II & 139 & 63.3 & & \\
\hline & III & 28 & 12.8 & & \\
\hline \multirow[t]{3}{*}{ Lymph node invasion } & No & 92 & 41.6 & 0.077 & 0.079 \\
\hline & $1-3$ & 43 & 19.6 & & \\
\hline & $>3$ & 115 & 38.8 & & \\
\hline
\end{tabular}

*: Correlation Coefficient; <0.1: poor correlation, $0.1-0.3$ : low correlation, 0.3-0.5: moderate correlation, $>0.5$ strong correlation 
rho $=0.077$, tau Kendall $=0.079$. We also found that there was no significant relationship between Ki-67 and tumor size (Spearman rho $=0.558$, tau Kendall $=0.548)$.

\section{DISCUSSION}

The current study was analytical-descriptive study and enrolled 220 patients with primary BC admitted to oncology clinic of Tabriz University of Medical Sciences. We evaluated the relationship between Ki67 expression and the clinicopathologic aspects, including the levels of ER and PR, lymph node status, and the grades and sizes of tumors in our breast cancer patients. We observed that Ki-67 were positive in $53 \%$ of patients and $24 \%$ of them had Ki-67 expression in more than $15 \%$ of tumor cells. No significant relationship was found between Ki-67 and either the patient's age or the size and grade of the tumors. However, there was a marginally significant relationship between the lymph node status and Ki-67 expression. Although there was a significant relationship between estrogen receptor (ER) and progesterone receptor (PR) with Ki-67 status, a reliable estimation of prognostic factors in $\mathrm{BC}$ patients is still required for the selection of the optimal therapeutic strategy.

In recent years, evidence has shown that the Ki-67 labeling index is an independent prognostic factor for the survival and recurrence of tumors. These studies examined more than 4600 cases and proved that Ki67 labeling index is a significant prognostic factor ${ }^{17}$. De Azambuja (2007) retrieved the disease-free survival (DFS) data from 46 studies and confirmed that high Ki-67 levels conferred a worse prognosis in the studied cohorts ${ }^{18}$. A number of adjuvant trials did not support the predictive role for the benefit of applying chemotherapy over endocrine treatment alone in patients with high tumor Ki-67 expression ${ }^{19}$. However, it is well-documented that higher levels of the proliferation marker Ki-67 are significantly associated with poor survival, high relapse and mortality rate ${ }^{20,21}$.

A few studies revealed a significant association between the pre-therapy Ki-67 and histological grade of tumors and an inverse association between Ki-67 with ER status ${ }^{22}$. A significant relationship was reported for Ki-67 and other tumor markers (ER, PR), which showed that increased Ki-67 levels were correlated with increased tumor grades ${ }^{22,23}$. However, in a review by Yerushalmi (2010), it was suggested that further studies are required before any recommendations can be made about using the relationship of tumor grade and Ki-67 ${ }^{19}$. In our study, however, no significant correlation was observed between tumor grade and Ki-67 levels (The Spearman rho $=0.570$, tau Kendall $=0.568$ ).

In a survey by Altintas (2009), the correlation between Ki-67and other biologic markers used and it was found that highly proliferative lesions were more likely to be ER negative and PR negative ${ }^{7}$. In another study, there was a significant negative relationship between Ki-67 levels and the expression of estrogen and progesterone receptors ${ }^{4}$. Additionally, Bouzubar (1989) suggested that, conversely, although the Ki-67 status of breast tumors and their percentage are not correlated with the ER status of BC, ERpositive tumors contain a slightly higher proportion of Ki-67 negative cells than ER-negative tumors ${ }^{24}$. We observed a significant correlation between Ki-67 and ER $(P=0.05)$ and a marginally significant correlation with PR $(P=0.07)$ by Mann-Whitney $U$ test. Our findings showed that there was a negative correlation between Ki-67and ER and PR.

In another study, a significant correlation was observed between the median Ki-67 staining and patient age and tumor nuclear grade. Tumors from patients younger than 50 years showed a higher level of Ki67 than those of older patients ${ }^{25}$. Other than that, none of similar studies has shown significant correlation between Ki-67 and patient's age, which is consistent with our result ${ }^{4,26}$.

The status of the LN remains to be the most important determinant of the overall survival, and nodenegative BC patients have a favorable prognosis ${ }^{27-29}$. Molino et al. found that there was a positive relationship with nodal status, as node negative tumors are more likely to have a low proliferation index ${ }^{4}$. A correlation between the histological grade of malignancy of breast tumors and their Ki-67 status was previously reported and no significant association was observed between tumor size, LN status, patient's age, ER, and Ki-67 status ${ }^{24}$. Although large tumors often contained an increased number of Ki-67 positive cells (up to $20 \%)^{24}$, some events showed a positive association between Ki-67 staining and tumor size, in which, the smaller tumors had lower Ki-67 values, and the larger tumors $(>2 \mathrm{~cm}$ ) were associated with poorer prognosis ${ }^{4,27}$.

\section{CONCLUSIONS}

Globally, breast cancer is the most common cancer in terms of incidence and mortality in women, which raises a social problem and a threat to the women health community. The recent molecular biology research provide useful information for personalized treatments. Therefore, patients will have more chance 
to choose a suitable therapy, which can prevent complications. Inaccessibility to molecular biology techniques among the communities with financial difficulty has led to the limited exploitation of these methods in treatment.

Nevertheless, IHC is an inexpensive and practical method that can be performed in all communitybased laboratories and can provide useful information about the morphologic and topographic traits of the tumors. It is an appropriate method to use in prognosis determination and the selection of proper therapy protocol. In other words, the correlation between the prognostic factor, Ki-67, and other prognostic and predictive factors assure the physicians a better management in patients with BC. In conclusion, in this study, we found that there is a significant relationship between Ki-67and ER and a marginally significant relationship between Ki-67 with PR and LN. However, no significant relationship was observed between Ki67 and patient's age, size and grade.

\section{ABBREVIATIONS}

BC: Breast Cancer

DFS: disease-free survival

ER: Estrogen Receptor

IHC: Immuno Histo-Cytochemistry

Ki-67: The Ki-67 protein (also known as MKI67) is a cellular marker for proliferation.

LN: Lymph Node

PR: Progesterone Receptor

\section{COMPETING INTERESTS}

The authors report no conflicts of interest in this work.

\section{AUTHORS' CONTRIBUTIONS}

TAK, and IAK: substantial contributed to conception and design of the study, acquisition of data, analysis and interpretation of data, drafted and wrote the article, did the final approval of the manuscript, agreed to be accountable for all aspects of the work in ensuring that questions related to the accuracy or integrity of any part of the work are appropriately investigated and resolved.

ZF: substantial contributed to conception and design of the study, revised the article, did the final approval of the manuscript, agreed to be accountable for all aspects of the work in ensuring that questions related to the accuracy or integrity of any part of the work are appropriately investigated and resolved.

RD: substantial contributed to conception and design of the study, analysis and interpretation of data, revised the article, did the final approval of the manuscript, agreed to be accountable for all aspects of the work in ensuring that questions related to the accuracy or integrity of any part of the work are appropriately investigated and resolved.

All authors read and approved the manuscript.

\section{ACKNOWLEDGMENTS}

This research study has been supported by Hematology and Oncology Research Center, as Medical Doctoral thesis of Dr. Zhaleh Faham (confirmed ID: 5/4/9587). This study was reviewed and approved by the ethics committee of Tabriz University of Medical Sciences. All patients enrolled in the study provided written informed consent forms.

\section{REFERENCES}

1. of Disease Cancer C Global B. Global, Regional, and National Cancer Incidence, Mortality, Years of Life Lost, Years Lived With Disability, and Disability-Adjusted Life-years for 32 Cancer Groups, 1990 to 2015: A Systematic Analysis for the Global Burden of Disease Study. JAMA Oncol. 2017;3(4):52448. Available from: 10.1001/jamaoncol.2016.5688.

2. Akbari ME, Sayad S, Sayad S, Khayamzadeh M, Shojaee L, Shormeji Z, et al. Breast Cancer Status in Iran: Statistical Analysis of 3010 Cases between 1998 and 2014. Int J Breast Cancer. 2017;2017:2481021. Available from: 10.1155/2017/2481021.

3. Arens N, Bleyl U, Hildenbrand R. HER2/neu, p53, Ki67, and hormone receptors do not change during neoadjuvant chemotherapy in breast cancer; 2005. Available from: 10.1007/s00428-005-1244-0;https://link.springer.com/ article/10.1007/s00428-005-1244-0.

4. Molino A, Micciolo R, Turazza $M$, Bonetti $F$, Piubello $Q$, Bonetti $A$, et al. Ki-67 immunostaining in 322 primary breast cancers: associations with clinical and pathological variables and prognosis. Int J Cancer. 1997;74(4):4337. Available from: 10.1002/(SICI)1097-0215(19970822)74: 4<433::AID-IJC12>3.0.CO;2-A.

5. Lu X, Gu Y, Ding Y, Song W, Mao J, Tan J, et al. Correlation of ER, PgR, HER-2/neu, p53, and VEGF with clinical characteristics and prognosis in Chinese women with invasive breast cancer. Breast J. 2008;14(3):308-10. Available from: 10.1111/j.15244741.2008.00583.x.

6. Roy SS, Vadlamudi RK. Role of estrogen receptor signaling in breast cancer metastasis. Int J Breast Cancer. 2012;2012:654698. Available from: 10.1155/2012/654698.

7. Altintas $S$, Lambein K, Huizing MT, Braems G, Asjoe FT, Hellemans $\mathrm{H}$, et al. Prognostic significance of oncogenic markers in ductal carcinoma in situ of the breast: a clinicopathologic study. Breast J. 2009;15(2):120-32. Available from: 10.1111/j.1524-4741.2009.00686.x.

8. Baek JM, Chae BJ, Song BJ, Jung SS. The potential role of estrogen receptor $\beta 2$ in breast cancer. Int J Surg. 2015;14:17-22. Available from: 10.1016/j.ijsu.2014.10.007.

9. Elebro $K$, Borgquist $S$, Rosendahl $A H$, Markkula $A$, Simonsson $M$, Jirstrom K. High Estrogen Receptor $\beta$ Expression Is Prognostic among Adjuvant Chemotherapy-Treated Patients-Results from a PopulationBased Breast Cancer Cohort; 2017. Available from: https://doi.org/10.1158/1078-0432.CCR-16-1095; http: //clincancerres.aacrjournals.org/content/23/3/766.abstract.

10. Kontzoglou K, Palla V, Karaolanis G, Karaiskos I, Alexiou I, Pateras I, et al. Correlation between Ki67 and breast cancer prognosis. Oncology. 2013;84(4):219-25. Available from: $10.1159 / 000346475$. 
11. Juríková M, Danihel, Polák, Varga I. Ki67, PCNA, and MCM proteins: markers of proliferation in the diagnosis of breast cancer. Acta Histochem. 2016;118(5):544-52. Available from: 10.1016/j.acthis.2016.05.002.

12. cSahin S, Gönül II, Çakir A, Seçkin S, Uluoğlu Ö. Clinicopathological Significance of the Proliferation Markers Ki67, RacGAP1, and Topoisomerase 2 Alpha in Breast Cancer. Int J Surg Pathol. 2016;24(7):607-13. Available from: 10.1177/ 1066896916653211.

13. Ellis MJ, Suman VJ, Hoog J, Goncalves R, Sanati S, Creighton $\mathrm{CJ}$, et al. Ki67 Proliferation Index as a Tool for Chemotherapy Decisions During and After Neoadjuvant Aromatase Inhibitor Treatment of Breast Cancer: Results From the American College of Surgeons Oncology Group Z1031 Trial (Alliance). J Clin Oncol. 2017;35(10):1061-9. Available from: 10.1200/JCO.2016.69.4406.

14. Carbognin L, Sperduti I, Fabi A, Dieci MV, Kadrija D, Griguolo $G$, et al. Prognostic impact of proliferation for resected early stage 'pure' invasive lobular breast cancer: cut-off analysis of Ki67 according to histology and clinical validation. Breast. 2017;35:21-6. Available from: 10.1016/j.breast.2017.06.005.

15. Ellis IO, Lee AH, Pinder SE, Rakha EA. Tumors of the breast. Flecher CDM, editor. Philadelphia: Elsevier; 2014

16. Tan PH, Ellis IO. Myoepithelial and epithelial-myoepithelial, mesenchymal and fibroepithelial breast lesions: updates from the WHO Classification of Tumours of the Breast 2012. J Clin Pathol. 2013;66(6):465-70. Available from: 10.1136/jclinpath2012-201078.

17. Scholzen T, Gerdes J. The Ki-67 protein: from the known and the unknown. J Cell Physiol. 2000;182(3):311-22. Available from: 10.1002/(SICI)1097-4652(200003)182:3<311::AIDJCP1>3.0.CO;2-9.

18. de Azambuja E, Cardoso F, Jr GdC, Colozza M, Mano MS Durbecq V, et al. Ki-67 as prognostic marker in early breast cancer: a meta-analysis of published studies involving 12,155 patients. Br J Cancer. 2007;96(10):1504-13. Available from: 10.1038/sj.bjc.6603756.

19. Yerushalmi R, Woods R, Ravdin PM, Hayes MM, Gelmon KA Ki67 in breast cancer: prognostic and predictive potential. Lancet Oncol. 2010;11(2):174-83. Available from: 10.1016/ S1470-2045(09)70262-1.

20. Liu Y, Zhang X, Yu F, Liu J, Zhang M, Zhang S, et al. [Prognostic significance of $\mathrm{Ki}-67$ expression before and after neoadju- vant chemotherapy in different biological breast cancer phenotypes]. Zhonghua Zhong Liu Za Zhi. 2014;36(9):671-6.

21. Matsubara N, Mukai H, Fujii S, Wada N. Different prognostic significance of Ki-67 change between pre- and postneoadjuvant chemotherapy in various subtypes of breast cancer. Breast Cancer Res Treat. 2013;137(1):203-12. Available from: 10.1007/s10549-012-2344-6.

22. Petrelli F, Viale G, Cabiddu M, Barni S. Prognostic value of different cut-off levels of Ki- 67 in breast cancer: a systematic review and meta-analysis of 64,196 patients. Breast Cancer Res Treat. 2015;153(3):477-91. Available from: 10.1007/s10549 015-3559-0

23. Chen $\mathrm{Q}, \mathrm{Wu} \mathrm{K}$. [Research progress on the clinical value of Ki-67 in breast cancer and its cut-off definition]. Zhonghua Wai Ke Za Zhi. 2015;53(8):634-7.

24. Bouzubar N, Walker KJ, Griffiths K, Ellis IO, Elston CW, Robertson JF, et al. Ki67 immunostaining in primary breast cancer: pathological and clinical associations. $\mathrm{Br} J$ Cancer 1989;59(6):943-7. Available from: 10.1038/bjc.1989.200.

25. Sahin $A A$, Ro J, Ro JY, Blick $M B$, el Naggar $A K$, Ordonez NG, et al. Ki-67 immunostaining in nodenegative stage $\mathrm{I} / \mathrm{II}$ breast carcinoma. Significant correlation with prognosis. Cancer. 1991;68(3):549-57. Available from: 10.1002/1097-0142(19910801)68:3<549:: AID-CNCR2820680318>3.0.CO;2-J.

26. Kim EK, Noh WC, Han W, Noh DY. Prognostic significance of young age ( $<35$ years) by subtype based on ER, PR, and HER2 status in breast cancer: a nationwide registry-based study. World J Surg. 2011;35(6):1244-53. Available from: 10.1007/s00268-011-1071-1.

27. Kuru B, Camlibel M, Gulcelik MA, Alagol H. Prognostic factors affecting survival and disease-free survival in lymph nodenegative breast carcinomas. J Surg Oncol. 2003;83(3):167-72. Available from: 10.1002/jso.10264.

28. Bonneau C, Bendifallah S, Reyal F, Rossi L, Rouzier R. Association of the number of sentinel lymph nodes harvested with survival in breast cancer; 2015. Available from: 10.1016/j.ejso.2014.11.004; https://www.ejso.com/article/ S0748-7983(14)01202-5/fulltext.

29. Yang J, Long Q, Li H, Lv Q, Tan Q, Yang X. The value of positive lymph nodes ratio combined with negative lymph node count in prediction of breast cancer survival. J Thorac Dis. 2017;9(6):1531-7. Available from: 10.21037/jtd.2017.05.30. 